\title{
How do energy balance-related behaviors cluster in adolescents?
}

\begin{abstract}
Objective: To delineate the clustering of energy balance-related behaviors in adolescents, and investigate whether these behaviors are associated with the household socioeconomic status and parental education level. Methods: Two cross-sectional studies assessed information on sedentary behavior, physical activity, sugar-sweetened beverages and fruit and vegetable consumption, and sleep duration by self-reported questionnaires in adolescents (12.5-17.5 years old) from Maringá/Brazil (BRACAH study; $\mathrm{n}=682$ ), and ten European cities (HELENA study; $\mathrm{n}=1252$ ) from nine different countries. Gender-specific cluster analyses were performed separately for each study, applying a combination of hierarchical and non-hierarchical methods. Results: Girls showed equivalent behaviors: Sedentary; Active; Unhealthy Eating; Healthy Eating; while boys differed (Brazilian: Sedentary; Active; Healthy Eating; European: Sedentary; Healthy; Unhealthy Eating). In Brazil, we found no association between socioeconomic status or parental education. In European girls, the high socioeconomic status and both parents' university degree were associated with Healthy Eating. In European boys, the high socioeconomic status was associated with Unhealthy Eating, and the mothers' university degree was associated with the Healthy cluster. Conclusions: Adolescents show Sedentary behavior, regardless of their sex, country of origin or socioeconomic condition.
\end{abstract}

Keywords: cluster analysis; energy balance-related behaviors; adolescents; socioeconomic status; parental education.

\section{INTRODUCTION}

WHO defines adolescents as people between 10 to 19 years of age (WHO 2015). Adolescence is a formative period in life that involves numerous physiological and psychological modifications, influencing nutritional needs and behaviors that directly affect lifelong health (Viner et al. 2012). The early establishment of healthy energy balance-related behaviors (EBRB) is considered to be an important precursor of the prevention of disorders such as obesity and cardiovascular diseases (Kavey RE 2003). Nonetheless, unhealthy EBRB (e.g. sedentary behavior, unhealthy diet, and sleep deprivation) are highly prevalent in youth (Johnson et al. 2009). For instance, almost 50\% of American adolescents showed at least one of these EBRB (Johnson et al. 2009). Additionally, more than $40 \%$ of adolescents in Finland, Portugal, Belgium, the United States, and Brazil exceed the screen time recommendation of a maximum of two hours per day (Rey-Lopéz JP 2011). Furthermore, fruit and vegetable consumption among adolescents at this age is below the recommendation, regardless of country of origin or income status (Krolner et al. 2011).

EBRB are not isolated as they tend to cluster towards healthier or unhealthier combinations (Leech et al. 2014). For example, sedentary behavior is associated with the high consumption of energy-dense foods and sugar-sweetened beverages as well as a low fruit and vegetable intake (Rodenburg et al. 2013). It seems that there are possible synergistic effects of EBRB on health (Santaliestra-Pasias et al. 2015). Consequently, there is increasing interest in analyzing the clustering of these behaviors to identify the populations that are at higher risk of developing chronic diseases (Leech et al. 2014). Furthermore, it has been observed that adolescents' EBRB are associated with economic and social factors within countries 
(Viner et al. 2012). Nevertheless, there is no clear consensus regarding socioeconomic status as a determinant of these behaviors, given that such an association may be attributed to the demographic context and characteristics of the studied populations (Seabra et al. 2008). The HELENA consortium previously published a description of EBRB clusters (Ottevaere et al. 2011). However, this article disregarded gender differences and sleep duration. Sleep duration is crucial for health, and in adolescents, insufficient sleep is a risk factor of chronic diseases (Nascimento-Ferreira et al. 2015). Moreover, little is known about gender differences in and the socioeconomic correlates of EBRB across different continents such as South America and Europe (Elgar et al. 2015). Hence, we aim to describe the clustering of several EBRB in adolescents in Brazil and Europe. Second, we aim to investigate whether these identified clusters are associated with socioeconomic status and parental education level.

\section{METHODS}

\section{Study Design and Population}

Data from the Brazilian Cardiovascular Adolescent Health (BRACAH) study were collected in 2007 in the city of Maringá, Brazil, which had approximately 330,000 inhabitants (15.6\% of whom were adolescents). A total of 682 adolescents (14.0-17.5 years old; 54.25\% girls) who were enrolled in public and private schools were selected using random sampling and evaluated on a broad range of health-related behaviors and cardiovascular risk factors. The total sample size $(\mathrm{n}=991)$ and the methodology of this study were described previously (Moraes et al. 2011). A formal request to conduct this survey was submitted and accepted by the boards of all participants' schools. This study was approved by the Ethics Committee on Research Involving Human Participants of the University Center of Maringá, and authorized by the Ethics Committee on Research Projects of the University of São Paulo, in accordance with Brazilian law. Written informed consent was obtained from participants' parents/guardians, and all research subjects agreed to participate in the study (Moraes et al. 2011).

The Healthy Lifestyle in Europe by Nutrition in Adolescence (HELENA) study is a multicenter cross-sectional study based on a random sample of clusters of European adolescents (12.5-17.5 years old) who were tested on a wide range of nutrition and health-related parameters using standardized procedures (Moreno et al. 2008). The data were collected in 2006 and 2007 in ten European cities (Athens, Greece, Dortmund, Germany, Ghent, Belgium, Heraklion, Crete, Lille, France, Pecs, Hungary, Rome, Italy, Stockholm, Sweden, Vienna, Austria, and Zaragoza, Spain). A detailed description of the HELENA sampling and recruitment strategies, harmonization processes, data collection procedures, analytic strategies, and quality control activities was published elsewhere (Moreno et al. 2008). After receiving complete information about the aims and methods of the study, all parents/guardians signed an informed consent form, and the adolescents agreed to participate in the study (Moreno et al. 2008). The protocol was approved by the Human Research Review Committees of the centers involved. In HELENA, 3528 adolescents were recruited. However, only 1252 (52.72\% girls) of them completed the food frequency questionnaire and provided all the information related to sedentary behavior, physical activity, sleeping duration, socioeconomic status and parental education level. Therefore, just these 1252 adolescents with complete information were included in the present analysis. 
The EBRB were assessed using self-report questionnaires in both studies.

Individual ExposuresThe questionnaire that was employed to assess sedentary time was found to be a reliable tool for use with adolescents (Rey-Lopez et al. 2012). This questionnaire included items to determine the amount of time spent on habitual screen-based activities (e.g., television, the Internet, video games) in daily minutes. In both studies, the questionnaire included questions such as "During weekdays, how many hours do you usually spend watching television?" - "During weekdays, how many hours do you usually spend on computers?" - "During weekdays, how many hours do you usually spend playing video games?" The same information was collected for weekend days. The average time spent per day on these activities was calculated (separately for the week and the weekend).

Physical activity in the last week was assessed using an adapted version of the International Physical Activity Questionnaire (IPAQ-A). The IPAQ was originally developed to allow participants to self-report their physical activity and inactivity and is a valid and reliable tool for use with European adults (15-69 years) (Craig et al. 2003). To adapt this questionnaire for use with adolescents, questions about physical activity "at work" were replaced by "at school", and activities in "the household domain" were shortened. To avoid over-reporting, the intensity of physical activity was adapted (Craig et al. 2003). The IPAQ-A was validated with accelerometer data (an objective measure) in adolescents (Hagstromer et al. 2008).

Sugar-sweetened beverage and fruit and vegetable consumption were assessed using a qualitative food frequency questionnaire (FFQ) that was adapted from the Health Behavior in School Children, which has been tested for reliability and validity (Vereecken and Maes 2003). This FFQ was administered in the classroom (under the supervision of a researcher). In the present study, we considered only the following food groups of interest: 1) Sugar-sweetened beverages; 2) Fruits; 3) Vegetables and greens. For example, one question on the FFQ asks, "How many times did you ingest fruit last week?" Therefore, research subjects were classified according to the number of servings consumed per week (Fewer than 1, 1-2, 3-4, $5-6$, and 7 servings/week). No specific amount was recorded. That is, only the frequency (weekly consumption) associated with each food group was taken into account.

Habitual sleep duration was estimated using another questionnaire with items, including "During weekdays: How many hours (and minutes) do you usually sleep?" and "During weekend days: How many hours (and minutes) do you usually sleep?" The reliability of this questionnaire was assessed in the HELENA study (with a subsample of 183 adolescents), showing almost perfect agreement ( 0.81 and 0.96 for weekdays and weekends, respectively). In a recent meta-analysis of the validity and reliability of sleep time questionnaires in children and adolescents, the authors identified a positive effect on the correlation coefficients of sleep duration between the questionnaires and accelerometers (objective measure) (Nascimento-Ferreira et al. 2015). 
The internal validity of all applied questionnaires were also tested in the BRACAH study (physical activity: Kappa coefficient $=0.91$; sedentary behavior: Kappa coefficient $=0.91$; FFQ: Kappa coefficient $=0.91$; sleep time: Kappa coefficient $=0.81$ and 0.96 on weekdays and weekend, respectively.

\section{Socioeconomic Status}

The Brazilian Criterion of Economic Classification was employed in the BRACAH study (ABEP 2006). This questionnaire assessed parental education level and the presence and number of domestic appliances, vehicles, and rooms in the household. With this information, a specific score (range 0-46) was computed and, the family was classified into one of the following seven categories: (A1 [the wealthiest], A2, B1, B2, C, D and E [the poorest]) (ABEP 2006). Finally, we grouped these categories into three socioeconomic levels: high (A1, A2, and B1), medium (B2 and C), and low (D and E).

In HELENA, the Family Affluence Scale, which measured the family structure, living conditions, and household goods, was used (Iliescu et al. 2008). This scale has been found to be a valid indicator of socioeconomic status in adolescents (Currie et al. 2008). The family was classified into one of three categories (range 0-8): A score from 0-3 indicates low socioeconomic status, 4-5 reflects medium socioeconomic status, and 6-8 indicates high socioeconomic status.

\section{Parental Education Level}

In both studies, data on mother and father education level were reported by the adolescents via a questionnaire (Iliescu et al. 2008). Although there are some differences in the classification of education level between Brazil and Europe, it was categorized into four groups (in the two studies): elementary education, lower secondary education, higher secondary education, and tertiary education.

\section{Potential Confounders}

The potential confounders that were considered in this study were survey center (in HELENA study) and school (both studies) as a contextual factor, and these factors were used in the adjusted models to control the variability of the associations between them. The variables age (in years), the Clusters, socioeconomic status and parental education level are individual factors.

\section{Statistical Analyses}

To identify clusters of similar behaviors, a combination of hierarchical and non-hierarchical clustering analyses was used (Fernandez-Alvira et al. 2013). Gender-specific cluster analyses were performed separately for each study due to significant differences in EBRB levels. Additionally, z-scores of all variables were calculated to standardize the dataset before clustering. This standardization prevents variables with larger ranges from contributing to cluster solutions to a greater extent than those with smaller ranges. Univariate and multivariate outliers (more than 3 standard deviations) were removed. First, we chose the Ward's method for applying hierarchical cluster analyzes based on the squared Euclidian distances (Ward 1963). Consequently, a comparison of several possible cluster solutions was performed. Using the resulting centers, nonhierarchical k-means cluster analyses were employed to fine-tune the 
preliminary hierarchical solution. ANOVA and Bonferroni post hoc tests were conducted to investigate the differences between clusters on all the variables.

The descriptive analyses are presented as the means (quantitative variables) or percentages (qualitative variables) and 95\% confidence intervals (95\% CI). We used Poisson multilevel regression models with fixed effects intercepts to estimate the prevalence ratios (PR), their respective confidence interval of 95\% (CI 95\%), and to analyze the relationship between each socioeconomic and parental education level with the clusters of EBRBs. We considered two levels of data organization: (i) clustering of EBRBs separately in each of the four groups (stratified by gender and region) and (ii) potential confounders (not shown) (Victora et al. 1997). The context variable that was used was the school. Homoscedasticity was graphically assessed in all regression models to meet the analyses' criteria. Furthermore, $p$-values of $\leq 0.20$ were adopted in the univariate analyses (Victora et al. 1997) since they were necessary to include variables in the multivariate analyses and the hierarchical model according to the aforementioned levels. Moreover, $p$-values $<0.05$ or those representing $>10 \%$ modification in the $\beta$ of any variable that was already in the model were considered to be significant.

The multilevel analyses were performed with two objectives: to test the associations between socioeconomic status, parental education level, and indicators of behaviors (z-scores) and to test the extent in which country-specific characteristics and contextual variables influence the associations between socioeconomic status, parental education level and clusters of EBRB. The associations between socioeconomic status and parental education level with the clusters were estimated using Poisson multilevel regression models with mixed effects intercepts, considering the Sedentary cluster as the reference category. We also verified if there were differences between the included and excluded adolescents in both of our samples.

Stata 12.0 (Stata Corp., College Station, TX, USA) was employed for the statistical calculations. All analyses were adjusted for the clustered nature of the sample using the "svy" set of commands and stratified by gender, as interactions between gender and the studied variables were observed $(p<0.001)$. To avoid the effect of multiple tests, the Bonferroni correction was applied to assess the associations between the clusters of EBRB and socioeconomic status $(p<0.017)$ and parental education level $(p<0.013)$.

\section{RESULTS}

\section{Characteristics of the Sample}

Table 1 presents the characteristics of the participants $(n=1,934)$ in the two cross-sectional studies. We have found no significant difference between the included and excluded adolescents form both of our samples.

In BRACAH, adolescents had a longer period of sedentary time and shorter periods of physical activity and sleeping than adolescents in the HELENA study. However, the mean fruit and vegetable consumption was higher in BRACAH than in HELENA study. 
Figure 1 highlights the estimated clusters of EBRB. The main outcome measures are on the horizontal axis and their related standard deviation z-scores on the vertical axis. Each cluster is numbered and named at the bottom of the graphic (different line colors), with their respective prevalence in parentheses. We identified four comparable clusters of EBRB for girls (Figures 1 A, B: 1. Sedentary; 2. Active; 3. Unhealthy Eating; 4. Healthy Eating), regardless of differences in prevalence. This wasn't the case in boys. Different clusters were found in BRACAH (Figure 1 C: 1. Sedentary; 2. Active; 3. Healthy Eating) and in HELENA (Figure 1 D: 1. Sedentary; 2. Healthy; 3. Unhealthy Eating).

Sedentary (Figures $1 \mathrm{~A}, \mathrm{~B}, \mathrm{C}, \mathrm{D}: \mathrm{n}^{\circ} 1$; represented by the purple line) was the only pattern identified in all four groups. It was characterized by z-scores above zero for screen time (e.g., watching TV, playing video games) and for sugar-sweetened beverage intake. Girls in both studies showed similar prevalence in the clusters. In Brazilian boys, the Sedentary cluster was dominant among the three clusters, whereas it was the least prevalent cluster in European boys.

The Active cluster (Figures $1 \mathrm{~A}, \mathrm{~B}, \mathrm{C}: \mathrm{n}^{\circ} 2$; represented by the green line) was determined by $\mathrm{z}$ scores above 1.5 for physical activity and negative z-scores for screen time. This cluster was observed in girls in both studies and in boys in BRACAH, but it was the least prevalent cluster in those three groups.

The Healthy cluster (Figure $1 \mathrm{D}: \mathrm{n}^{\circ} 2$; represented by the blue line) was exclusively identified in European boys with respect to low screen time and sugar-sweetened beverage intake and high physical activity, fruit and vegetable consumption, and sleep duration.

The Unhealthy Eating cluster (Figures 1 A, B, D: $n^{\circ} 3$; represented by the red line) was characterized by positive z-scores for sugar-sweetened beverage intake and negative z-scores for fruit and vegetable consumption and sleep duration. This Unhealthy Eating behavior was the most prevalent among the girls in the BRACAH study. Inversely, the Healthy Eating cluster (Figures $1 \mathrm{~A}, \mathrm{~B}: \mathrm{n}^{\circ} 4 ; \mathrm{C}^{\circ} \mathrm{n}^{\circ} 3$; represented by the pink line) was defined by positive z-scores for fruit and vegetable consumption and negative z-scores for sugar-sweetened beverage intake. The Healthy Eating cluster was the most prevalent cluster among girls in the HELENA study.

\section{Clusters of EBRB and Associations with Socioeconomic Status and Parental Education Level}

The clusters of EBRB by socioeconomic status and parental education level in BRACAH and HELENA studies are presented in Table 2. Furthermore, the $p$-values for these associations are provided in the supplementary material (Table 3). The clusters that were significantly associated with either socioeconomic status or parental education are shown in Table 3 with an asterisk, and Figures 2 and 3 illustrate their respective prevalence ratios (PR). In the BRACAH study, we did not identify any significant association between the clusters and socioeconomic status or parental education. However, in HELENA study, higher socioeconomic status (PR: 3.38; 95\% CI: 1.58 - 7.21) was associated with the Healthy Eating cluster among girls (Figure $2 \mathrm{~A}$ ), while higher socioeconomic status (PR: 2.78; 95\% CI: 1.28 - 6.04) was positively associated with the Unhealthy Eating cluster in boys (Figure 2 B). 
Regarding parental education, in the HELENA study, the mother's (PR: 6.98; 95\% CI: 2.55 19.09) and father's (PR: 6.48; 95\% CI: 2.26 - 18.62) university degree was significantly associated with the Healthy Eating cluster in girls (Figure 3A). Moreover, the mother's university degree (Figure 3B) was directly associated with the Healthy cluster (PR: 3.18 ; 95\% CI: 1.38 - 7.35) in European boys.

\section{DISCUSSION}

We identified four equivalent EBRB clusters for girls and three different clusters for boys. One of the major findings is that the Sedentary cluster (mainly characterized by a high level of screen time and low level of physical activity) was common across studies and genders.

It is notable that, although they lived in geographically distant regions, surrounded by distinct types of physical, economic, socio-cultural, and political environments (Delany-Moretlwe et al. 2015), girls in both studies had equivalent EBRB clusters (regardless of the distribution of their prevalence), and the most prevalent clusters were those that were primarily related to eating behavior (Unhealthy and Healthy). This similarity in dietary behavior is in line with previous studies (Ottevaere et al. 2011; Sabbe et al. 2008). Hill (Hill 2006) showed that girls tend to be more concerned with their appearance than boys because of the media's prominent role in setting "beauty ideals". Thus, they become more critical of their nutritional habits and pursue diets (that are not necessarily healthy) due to body image dissatisfaction (DjordjevicNikic and Dopsaj 2013). In boys, no such trend was analyzed. Most Brazilian boys were categorized in the Sedentary cluster, whereas most European boys were in the Healthy cluster. Regarding dietary intake, the observed clustering in the two studies diverged (Healthy Eating and Unhealthy Eating in boys from Brazil and Europe, respectively). These results among boys suggest that their behaviors may be more associated with individual-level (e.g., school and neighborhood) factors than those of girls (Sirard et al. 2013).

Even though adolescents from the HELENA study had a higher prevalence of healthier clusters (girls: $35.7 \%$ Healthy Eating; boys: $42.7 \%$ Healthy), in both studies and genders, other unhealthy-related behaviors occurred, and Sedentary was the only cluster found in all of them. This finding requires attention, as studies indicate that time spent on screen-based activities is associated with high energy intake (via energy-dense snacks and sugar-sweetened beverages) and less fruit and vegetable consumption, rather than low energy expenditure (Hobbs et al. 2014). Furthermore, the Active cluster (which was mainly characterized by z-scores above 1.5 for physical activity and negative z-scores for screen time) was the least prevalent cluster in girls in both studies and in boys in the BRACAH study.

Boys in the HELENA study did not show this Active pattern. However, only they had the Healthy cluster. This infrequent cluster suggests that several lifestyle trends do not always discriminate in the same direction. Previous studies demonstrated that teenagers have difficulty meeting multiple recommended health guidelines concurrently because people often adhere to one healthy behavior to compensate for other unhealthy habits (Ottevaere et al. 2011).

In terms of usual sleep duration, negative z-scores primarily were related to the Unhealthy Eating cluster. A recent study noted that sleep restriction increases the time available for eating and promotes 
fatigue, reducing engagement in physical activity (Thivel et al. 2015). In other research with adolescents, a later bedtime upregulated appetite, favoring higher energy intake (approximately $425 \mathrm{kcal} /$ day) in teenagers with a low sleep duration than in those with an adequate sleep time (Adamo KB 2013).

The results pertaining to the second goal, the characterization of the clustering of EBRB with socioeconomic status and parental education level, revealed that associations were different according to the study and gender. Unexpectedly, no significant association was found in the BRACAH study, while high socioeconomic status was associated with only two clusters in the HELENA study (Healthy Eating and Unhealthy Eating in girls and boys, respectively). This association between high socioeconomic status and Unhealthy Eating cluster is controversial, however previous studies also found difficulties while associating socioeconomic status with behaviors, particularly in adolescents (Beck CC 2011; Elgar et al. 2015; Ferrar and Golley 2015). This lack of an association in the BRACAH study could be due to several reasons, including that adolescents' behaviors are differently impacted by socioeconomic indicators and by peer-group influences (Michels et al. 2018), the BRACAH sample had an economic distribution that was more concentrated in the medium class (approximately 80\%), the methods used to estimate socioeconomic level in the two studies were distinct (Beck CC 2011), and the way that educational level is classified in Brazil is different from the European classification (ABEP 2006; Iliescu et al. 2008).

In the HELENA study, both parents' university degrees were significantly associated with the Healthy Eating in girls, and the mother's university degree was associated with the Healthy cluster in boys. These associations between higher parental education and adolescents' healthier behaviors seem to influence mediators such as parents' attitudes and beliefs, availability and accessibility, self-control, and knowledge, which are modifiable mediators that may hold promise for the development of obesity interventions (Ferrar and Golley 2015). We suppose that these associations were uncommon in Brazil because of the BRACAH's sample characteristics, with an important concentration of parents with upper secondary education (more than 40\%). Still, it emphasizes that improved education is essential to promote health (Gakidou et al. 2010).

\section{Limitations}

Our studies with Brazilian and European adolescents should be cautiously extrapolated to the corresponding overall populations; hence, we are taking into account geographic areas that have different peculiarities and environmental elements that may misrepresent the influence of socioeconomic status on clustering behaviors(Beck CC 2011). In addition, it is important to notice that we have considered only the weekly consumption of two food groups (fruits and vegetables and sugar-sweetened beverages) in our analysis since they are reported by the American Heart Association as components of the Ideal Cardiovascular Health in Children and Adolescents (Steinberger et al. 2016). Furthermore, data were obtained by self-report questionnaires, which also have bias such as social desirability and under/overreporting of information (Ottevaere et al. 2011). However, every assessment method comes with its own set of limitations, and no specific tool can be acknowledged as the best option (Corella and Ordovas 2015). Furthermore, questionnaires are frequently applied in centers with low financial resources, because they 
are more feasible and inexpensive than objective methods and are associated with relatively low participant burden (Murakami et al. 2016).

Despite a few methodological differences (e.g., socioeconomic questionnaire) between studies, one strength of our research is the possibility of comparing adolescents' EBRB between Brazil and some European countries. This is an innovative study with cluster analysis and their associations with socioeconomic factors in adolescents.

\section{Conclusions}

Adolescents show Sedentary behavior, regardless of their sex, country of origin or socioeconomic condition. Girls showed equivalent energy balance-related behaviors, while boys differed, suggesting that boys' behaviors are more associated with individual-level factors than those of girls. In this sense, it is highly important that public health policies target different interventions for girls and boys.

Family socioeconomic conditions are more associated with Europeans' than Brazilians' behaviors. However, both studies emphasize that improved education is essential to promote health and that identifying these energy-balanced behaviors may help to develop new interventions for the prevention of obesity.

\section{LIST OF ABBREVIATIONS}

- EBRB: Energy Balance-Related Behaviors

- BRACAH Study: Brazilian Cardiovascular Adolescent Health Study

- HELENA Study: Healthy Lifestyle in Europe by Nutrition in Adolescence Study

- IPAQ-A: International Physical Activity Questionnaire

- FFQ: Food Frequency Questionnaire

- PR: Prevalence Ratio

\section{COMPLIANCE WITH ETHICAL STANDARDS}

Data from the Brazilian Cardiovascular Adolescent Health (BRACAH) Study was approved by the Ethics Committee on Research Involving Human Participants of the University Center of Maringá, and authorized by the Ethics Committee on Research Projects of the University of São Paulo, in accordance with Brazilian law. The Healthy Lifestyle in Europe by Nutrition in Adolescence (HELENA) Study was approved by the Human Research Review Committees of all the European centers involved. Both studies were conducted according to the guidelines laid down in the Declaration of Helsinki, and a formal request to conduct the survey was submitted and accepted by the boards of all participant schools. Written informed consent was obtained from all adolescents as well as from their parents/guardians. 


\section{REFERENCES}

ABEP: The Brazilian Research Companies Association (2006). The Brazilian Criteria for Economic Classification (CCEB). http://www.abep.org/codigos-e-guias-da-abep. Accessed 30 June 2011.

Adamo KB WS, Belanger K, Chaput JP (2013) Later Bedtime is Associated with Greater Daily Energy Intake and Screen Time in Obese Adolescents Independent of Sleep Duration. vol 2. J Sleep Disord Ther.

Beck CC LA, Pitanga FJG. (2011) Indicadores antropométricos como preditores de pressão arterial elevada em adolescentes. vol 96. Arq Bras Cardiol.

Corella D, Ordovas JM (2015) Biomarkers: background, classification and guidelines for applications in nutritional epidemiology Nutr Hosp 31 Suppl 3:177-188 doi:10.3305/nh.2015.31.sup3.8765

Craig CL, Marshall AL, Sjostrom M, et al. (2003) International physical activity questionnaire: 12-country reliability and validity Med Sci Sports Exerc 35:1381-1395. doi:10.1249/01.mss.0000078924.61453

Currie C, Molcho M, Boyce W, Holstein B, Torsheim T, Richter M (2008) Researching health inequalities in adolescents: the development of the Health Behaviour in School-Aged Children (HBSC) family affluence scale. In: Soc Sci Med, vol 66. vol 6. England, pp 1429-1436. doi:10.1016/j.socscimed.2007.11.024

Delany-Moretlwe S, Cowan FM, Busza J, Bolton-Moore C, Kelley K, Fairlie L (2015) Providing comprehensive health services for young key populations: needs, barriers and gaps. In: J Int AIDS Soc, vol 18. vol 2(Suppl 1). p 19833

Djordjevic-Nikic M, Dopsaj M (2013) Characteristics of eating habits and physical activity in relation to body mass index among adolescents J Am Coll Nutr 32:224-233 doi:10.1080/07315724.2013.791149

Elgar FJ, Pfortner TK, Moor I, De Clercq B, Stevens GW, Currie C (2015) Socioeconomic inequalities in adolescent health 2002-2010: a time-series analysis of 34 countries participating in the Health Behaviour in School-aged Children study Lancet doi:10.1016/s0140-6736(14)61460-4

Fernandez-Alvira JM, De Bourdeaudhuij IS, Amika SV, et al. (2013) Clustering of energy balance-related behaviors and parental education in European children: the ENERGY-project Int J Behav Nutr Phys Act 10:5 doi:10.1186/1479-5868-10-5

Ferrar K, Golley R (2015) Adolescent Diet and Time Use Clusters and Associations With Overweight and Obesity and Socioeconomic Position Health Educ Behav doi:10.1177/1090198114560017

Gakidou E, Cowling K, Lozano R, Murray CJ (2010) Increased educational attainment and its effect on child mortality in 175 countries between 1970 and 2009: a systematic analysis. In: Lancet, vol 376. vol 9745. 2010 Elsevier Ltd, England, pp 959-974. doi:10.1016/s0140-6736(10)61257-3

Hagstromer M, Bergman P, De Bourdeaudhuij I, et al. (2008) Concurrent validity of a modified version of the International Physical Activity Questionnaire (IPAQ-A) in European adolescents: The HELENA Study Int J Obes (Lond) 32 Suppl 5:S42-48 doi:10.1038/ijo.2008.182

Hill AJ (2006) Motivation for eating behaviour in adolescent girls: the body beautiful Proc Nutr Soc 65:376384

Hobbs M, Pearson N, Foster PJ, Biddle SJ (2014) Sedentary behaviour and diet across the lifespan: an updated systematic review Br J Sports Med doi:10.1136/bjsports-2014-093754

Iliescu C, Beghin L, Maes L, et al. (2008) Socioeconomic questionnaire and clinical assessment in the HELENA Cross-Sectional Study: methodology Int J Obes (Lond) 32 Suppl 5:S19-25 doi:10.1038/ijo.2008.178

Johnson WD, Kroon JJ, Greenway FL, Bouchard C, Ryan D, Katzmarzyk PT (2009) Prevalence of risk factors for metabolic syndrome in adolescents: National Health and Nutrition Examination Survey (NHANES), 2001-2006 Arch Pediatr Adolesc Med 163:371-377 doi:10.1001/archpediatrics.2009.3

Kavey RE DS, Lauer RM, Atkins DL, Hayman LL, Taubert K. (2003) American Herat Association guidelines for primary prevention of atherosclerotic cardiovascular disease beginning in childhood. Circulation,

Krolner R, Rasmussen M, Brug J, Klepp KI, Wind M, Due P (2011) Determinants of fruit and vegetable consumption among children and adolescents: a review of the literature. Part II: qualitative studies Int J Behav Nutr Phys Act 8:112 doi:10.1186/1479-5868-8-112 
Leech RM, McNaughton SA, Timperio A (2014) The clustering of diet, physical activity and sedentary behavior in children and adolescents: a review Int J Behav Nutr Phys Act 11:4 doi:10.1186/14795868-11-4

Michels N, Vynckier L, Moreno LA, et al. (2018) Mediation of psychosocial determinants in the relation between socio-economic status and adolescents' diet quality Eur J Nutr 57:951-963 doi:10.1007/s00394-017-1380-8

Moraes AC, Delaporte TR, Molena-Fernandes CA, Falcao MC (2011) Factors associated with medicine use and self medication are different in adolescents Clinics (Sao Paulo) 66:1149-1155

Moreno LA, De Henauw S, Gonzalez-Gross M, et al. (2008) Design and implementation of the Healthy Lifestyle in Europe by Nutrition in Adolescence Cross-Sectional Study Int J Obes (Lond) 32 Suppl 5:S4-11 doi:10.1038/ijo.2008.177

Murakami H, Kawakami R, Nakae S, Nakata Y, Ishikawa-Takata K, Tanaka S, Miyachi M (2016) Accuracy of Wearable Devices for Estimating Total Energy Expenditure: Comparison With Metabolic Chamber and Doubly Labeled Water Method JAMA Intern Med 176:702-703 doi:10.1001/jamainternmed.2016.0152

Nascimento-Ferreira MV, Collese TS, de Moraes AC, Rendo-Urteaga T, Moreno LA, Carvalho HB (2015) Validity and reliability of sleep time questionnaires in children and adolescents: A systematic review and meta-analysis Sleep Med Rev 30:85-96 doi:10.1016/j.smrv.2015.11.006

Ottevaere C, Huybrechts I, Benser J, et al. (2011) Clustering patterns of physical activity, sedentary and dietary behavior among European adolescents: The HELENA study BMC Public Health 11:328 doi:10.1186/1471-2458-11-328

Rey-Lopez JP, Ruiz JR, Ortega FB, et al. (2012) Reliability and validity of a screen time-based sedentary behaviour questionnaire for adolescents: The HELENA study Eur J Public Health 22:373-377 doi:10.1093/eurpub/ckr040

Rey-Lopéz JP V-RG, Bueno G, Moreno LA. (2011) Chapter 20: Sedentary Behaviors and Obesity in Children and Adolescents. In: 2. SSoEaPH (ed) Epidemiology of Obesity in Children and Adolescents. pp 367-376

Rodenburg G, Oenema A, Kremers SPJ, van de Mheen D (2013) Clustering of diet- and activity-related parenting practices: cross-sectional findings of the INPACT study $\% \mathrm{U}$ http://www.ijbnpa.org/content/10/1/36 International Journal of Behavioral Nutrition and Physical Activity 10\%@1479-5868:36

Sabbe D, De Bourdeaudhuij I, Legiest E, Maes L (2008) A cluster-analytical approach towards physical activity and eating habits among 10-year-old children Health Educ Res 23:753-762 doi:10.1093/her/cyl135

Santaliestra-Pasias AM, Mouratidou T, Reisch L, et al. (2015) Clustering of lifestyle behaviours and relation to body composition in European children. The IDEFICS study Eur J Clin Nutr 69:811816 doi:10.1038/ejen.2015.76

Seabra AF, Mendonca DM, Thomis MA, Anjos LA, Maia JA (2008) [Biological and socio-cultural determinants of physical activity in adolescents]. In: Cad Saude Publica, vol 24. vol 4. Brazil, pp 721-736

Sirard JR, Bruening M, Wall MM, Eisenberg ME, Kim SK, Neumark-Sztainer D (2013) Physical activity and screen time in adolescents and their friends Am J Prev Med 44:48-55 doi:10.1016/j.amepre.2012.09.054

Steinberger J, Daniels SR, Hagberg N, et al. (2016) Cardiovascular Health Promotion in Children: Challenges and Opportunities for 2020 and Beyond: A Scientific Statement From the American Heart Association Circulation 134:e236-255 doi:10.1161/cir.0000000000000441

Thivel D, Isacco L, Aucouturier J, et al. (2015) Bedtime and Sleep Timing but not Sleep Duration Are Associated With Eating Habits in Primary School Children J Dev Behav Pediatr doi:10.1097/dbp.0000000000000131

Vereecken CA, Maes L (2003) A Belgian study on the reliability and relative validity of the Health Behaviour in School-Aged Children food-frequency questionnaire Public Health Nutr 6:581-588

Victora CG, Huttly SR, Fuchs SC, Olinto MT (1997) The role of conceptual frameworks in epidemiological analysis: a hierarchical approach Int J Epidemiol 26:224-227

Viner RM, Ozer EM, Denny S, Marmot M, Resnick M, Fatusi A, Currie C (2012) Adolescence and the social determinants of health Lancet 379:1641-1652 doi:10.1016/s0140-6736(12)60149-4

Ward J (1963) HIERARCHICAL GROUPING TO OPTIMIZE AN OBJECTIVE FUNCTION Journal of the American Statistical Association 58:236-\& doi:10.2307/2282967

WHO (2015) Core competencies in adolescent health and development for primary care providers: including a tool to assess the adolescent health and development component in pre-service education of health-care providers. 
Figure 1: clusters solutions according to the standard deviation (SD) z-scores on energy balancerelated behaviors (EBRB) for the Brazilian Cardiovascular Adolescent Health (BRACAH) and the Healthy Lifestyle in Europe by Nutrition in Adolescence (HELENA) studies. Brazil and Europe,2007. Clusters of EBRB are presented in different line colors. Clusters of EBRB are numbered, named, and with their respective prevalence (in parenthesis) at the bottom of each graphic.

Figure 2 A and B: Clusters of energy balance-related behaviors (EBRB) that were significantly associated with socioeconomic status and their respective prevalence ratio. Brazilian Cardiovascular Adolescent Health (BRACAH) and the Healthy Lifestyle in Europe by Nutrition in Adolescence (HELENA) studies. Brazil and Europe, 2007. Clusters of EBRB are indicated in different color boxes.

Figure 3 A and B: Clusters of energy balance-related behaviors (EBRB) that were significantly associated with parental education level and their respective prevalence ratio. Brazilian Cardiovascular Adolescent Health (BRACAH) and the Healthy Lifestyle in Europe by Nutrition in Adolescence (HELENA) studies. Brazil and Europe, 2007. Clusters of EBRB are indicated in different color boxes. 


\section{SD}

z-score

A) BRACAH GIRLS

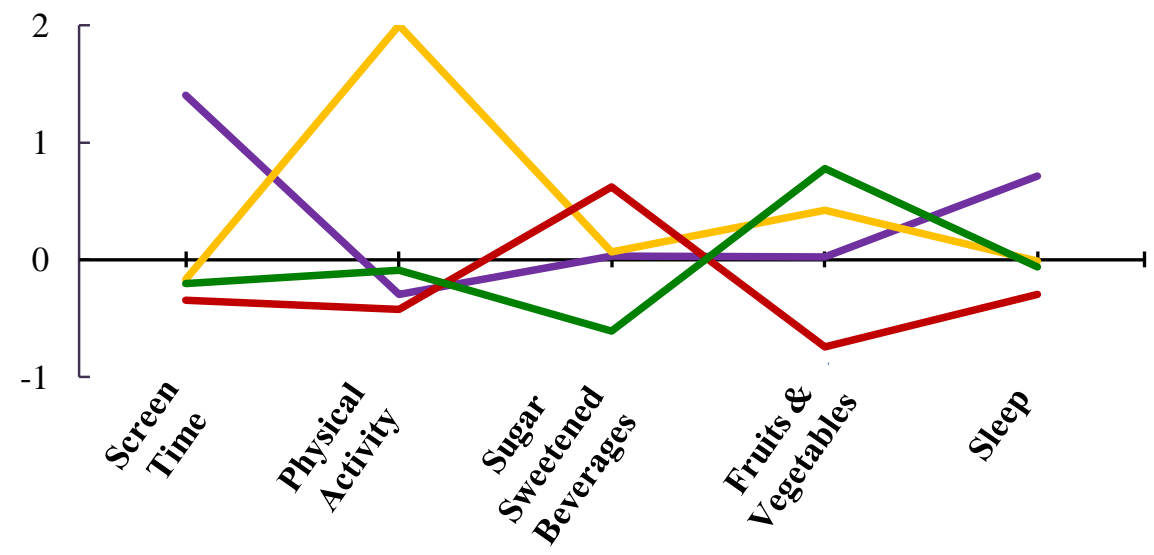

Clusters of EBRB (\%):

1. Sedentary $(\mathbf{1 7 . 8 \%})$

2. Active (14.5\%)

3. Unhealthy Eating (36.2\%)

4. Healthy Eating (31.5\%)

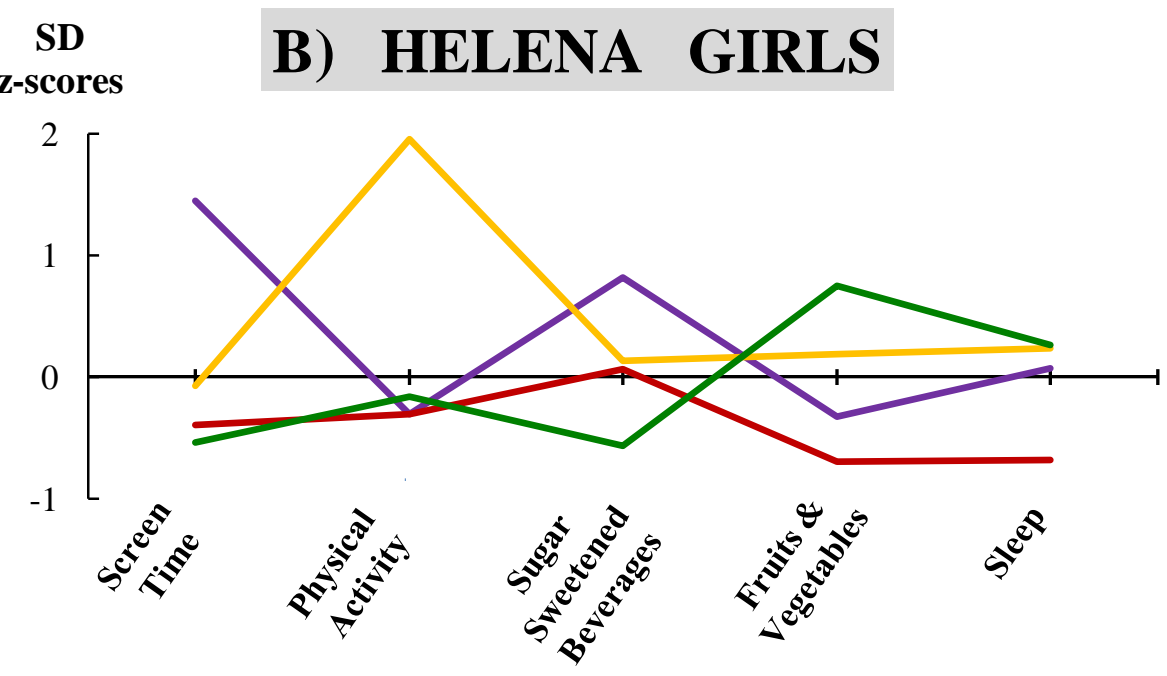

Clusters of EBRB (\%):

1. Sedentary $(\mathbf{2 0 . 8 \%})$

2. Active (15.9\%)

3. Unhealthy Eating (27.7\%)

4. Healthy Eating $(35.7 \%)$
SD

z-score

C) BRACAH BOYS

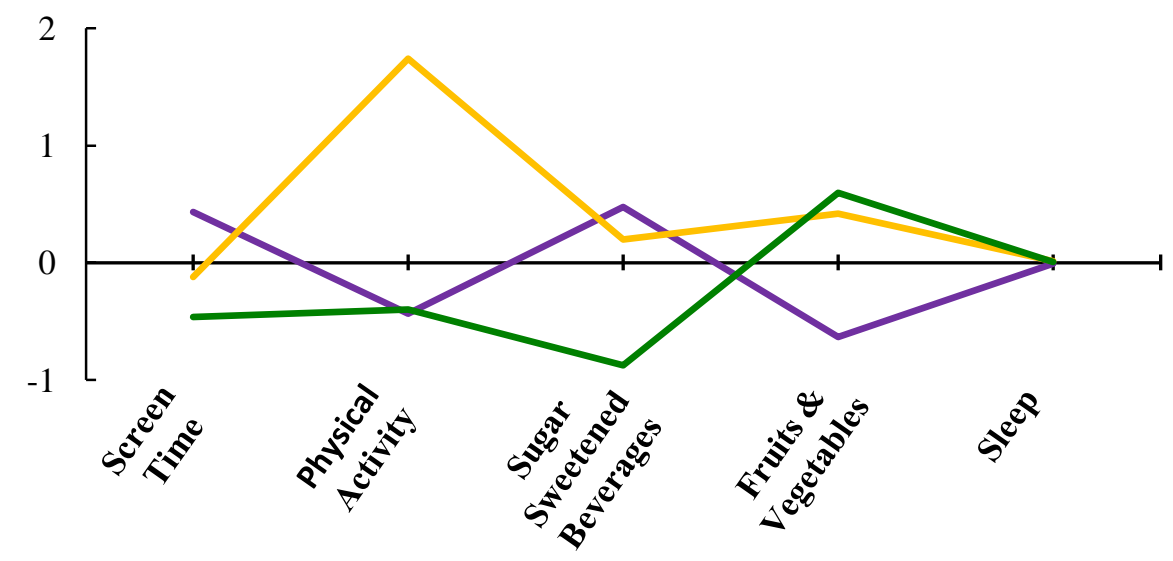

Clusters of EBRB (\%):

1. Sedentary $(44.7 \%)$
-3. Healthy Eating $(33.8 \%)$

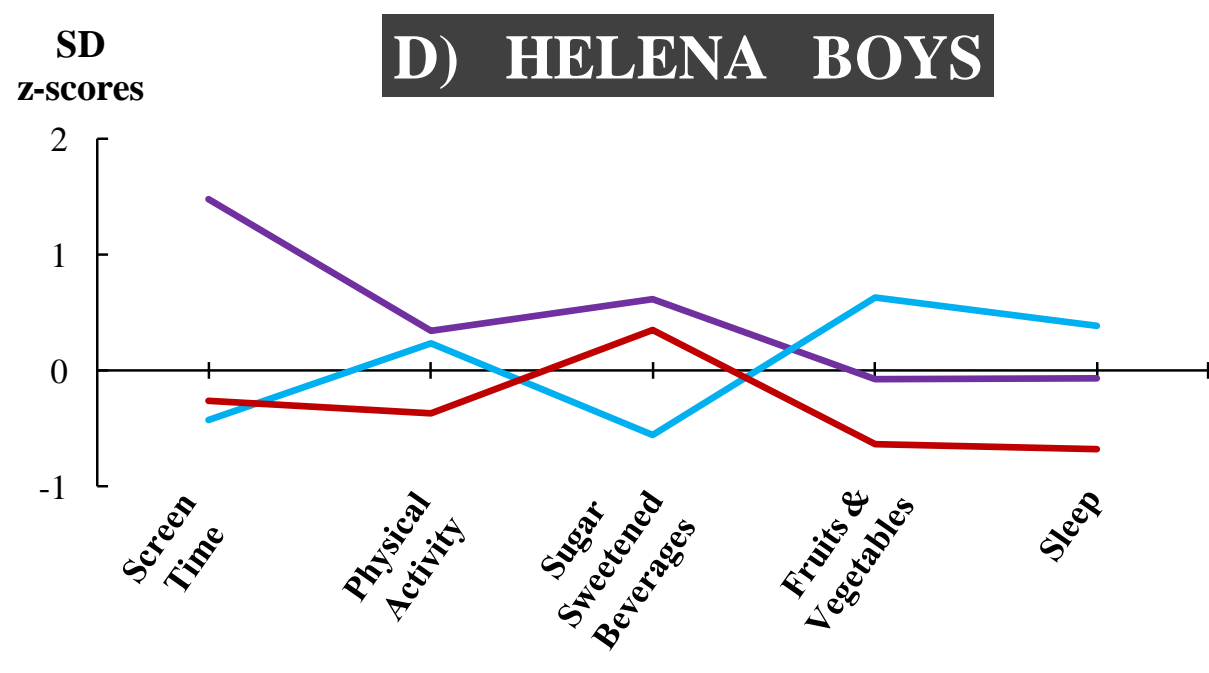

Clusters of EBRB (\%):

- 1. Sedentary (23.9\%)

2. Healthy $(42.7 \%)$

3. Unhealthy Eating (33.3\%) 


\section{A) HELENA GIRLS}

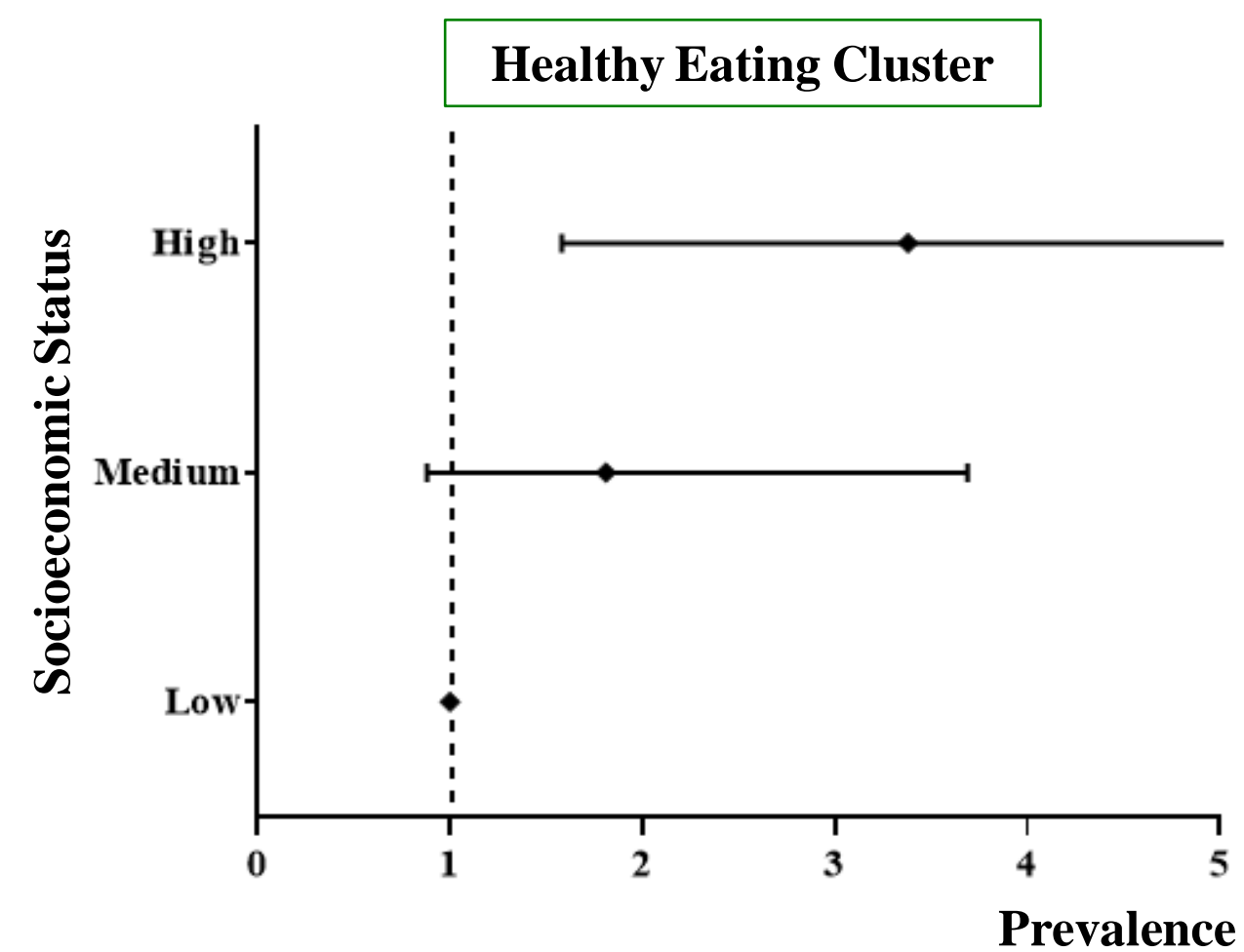

\section{B) HELENA BOYS}

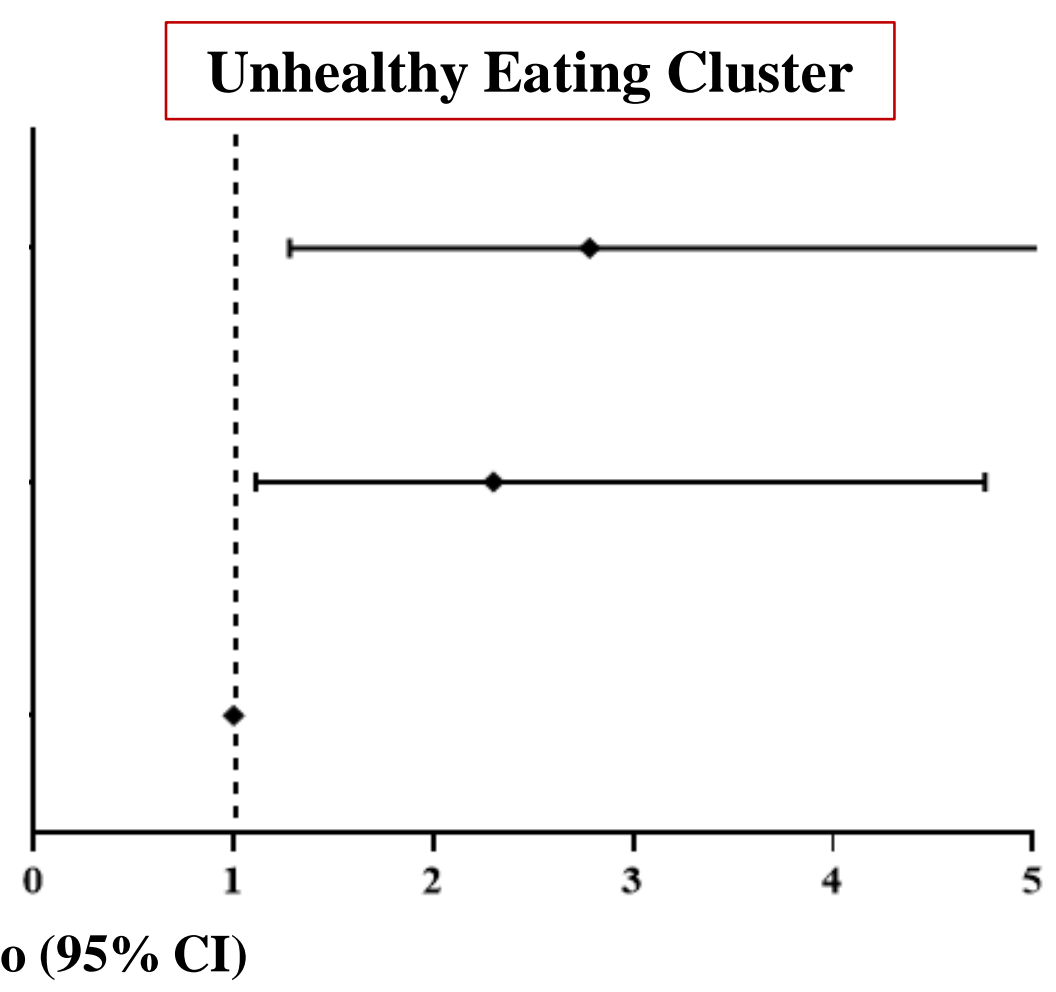




\section{A) HELENA GIRLS}

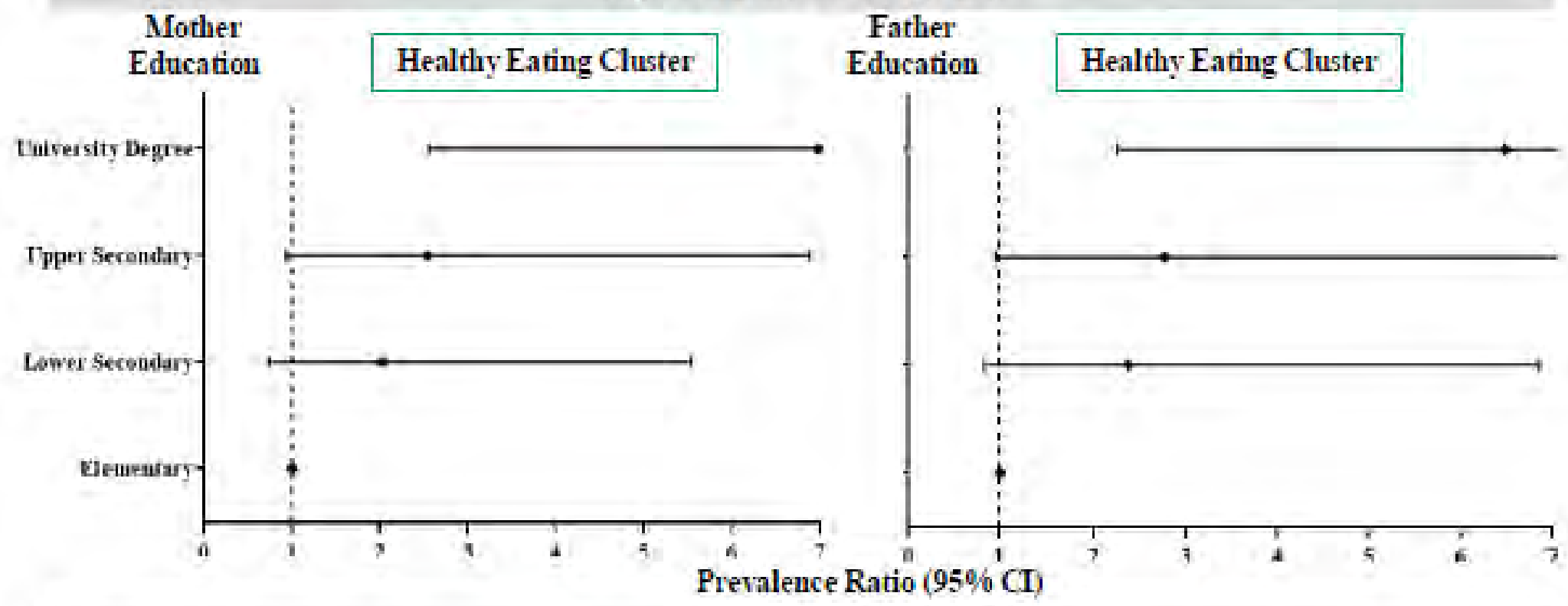

B) HELENA BOYS

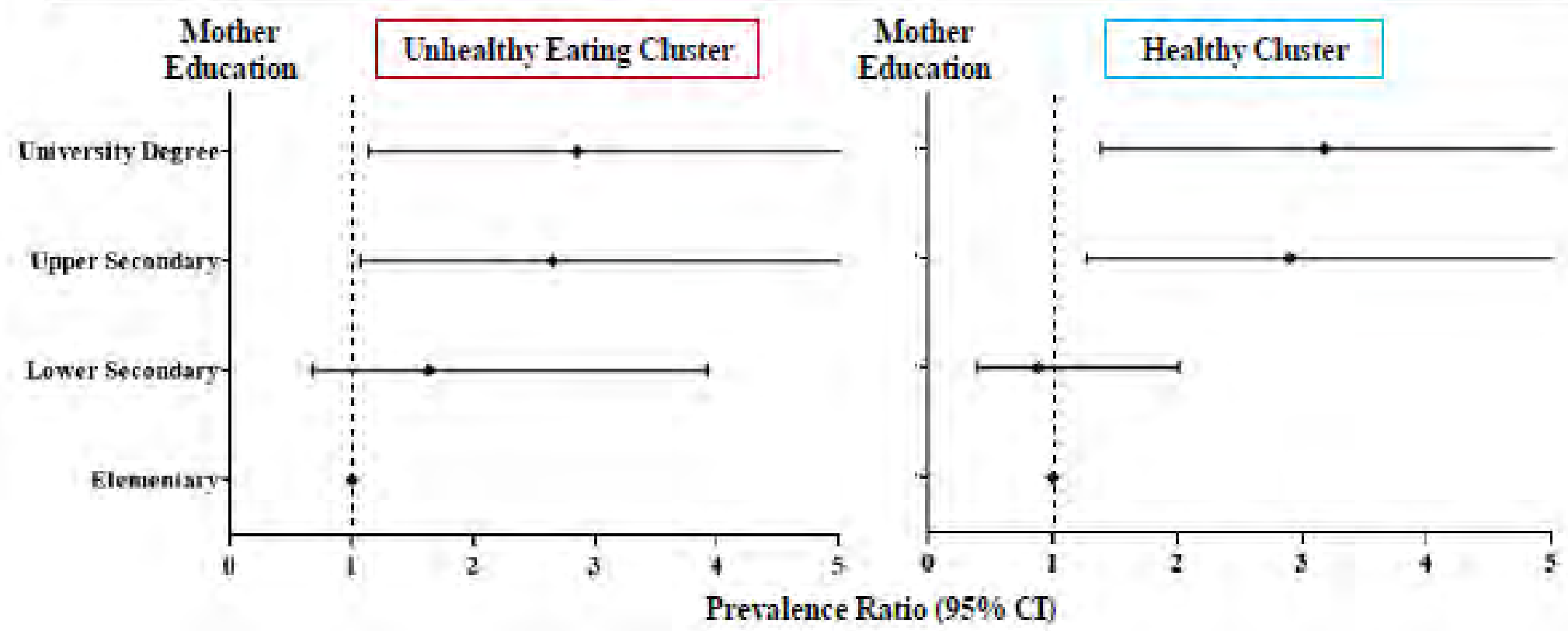


Table 1. Characteristics of the samples of adolescents from the Brazilian Cardiovascular Adolescent Health (BRACAH) and the Healthy Lifestyle in Europe by Nutrition in Adolescence (HELENA) studies ${ }_{-,}$. Brazil and Europe, 2007

\begin{tabular}{|c|c|c|c|c|}
\hline \multirow[t]{2}{*}{ Variables } & $\begin{array}{c}\text { BRACAH GIRLS } \\
(\mathrm{n}=370)\end{array}$ & $\begin{array}{l}\text { BRACAH BOYS } \\
(n=312)\end{array}$ & $\begin{array}{l}\text { HELENA GIRLS } \\
(\mathrm{n}=660)\end{array}$ & $\begin{array}{c}\text { HELENA BOYS } \\
(n=592)\end{array}$ \\
\hline & Mean* or \% (95\% CI) & Mean* or \% (95\% CI) & Mean* or \% (95\% CI) & Mean* or \% $(95 \%$ CI $)$ \\
\hline Age (years)* & $16.3(16.2-16.4)$ & $16.5(16.4-16.6)$ & $14.7(14.6-14.8)$ & $14.8(14.7-14.9)$ \\
\hline Sedentary time $(\min / \text { day })^{*}$ & $412.2(395.8-428.7)$ & $340.8(326.1-355.5)$ & $159.2(154.2-164.3)$ & $231.0(223.8-238.1)$ \\
\hline Physical activity (min/day)* & $45.0(40.9-49.0)$ & $58.1(52.4-63.7)$ & $53.1(51.0-55.3)$ & $67.3(64.6-70.5)$ \\
\hline Sweetened beverages ( $\geq 1$ serving/day) & $33.9(29.7-38.0)$ & $29.3(24.9-33.6)$ & $37.9(34.7-41.1)$ & $25.4(22.4-28.5)$ \\
\hline Fruit\&vegetable ( $\geq 1$ serving/day) & $47.0(36.8-48.9)$ & $36.8(32.2-41.4)$ & $36.6(31.5-41.6)$ & $32.1(28.0-38.4)$ \\
\hline Sleep (hours/day)* & $7.6(7.4-7.8)$ & $7.6(7.4-7.8)$ & $8.0(7.9-8.1)$ & $8.1(8.0-8.2)$ \\
\hline \multicolumn{5}{|l|}{ Socioeconomic status } \\
\hline Low & $9.2(6.2-12.2)$ & $4.3(2.0-6.6)$ & $10.5(8.1-13.0)$ & $10.2(7.7-12.8)$ \\
\hline Medium & $77.5(73.2-81.8)$ & $82.5(78.1-86.8)$ & $53.0(49.1-57.0)$ & $56.6(52.4-60.7)$ \\
\hline High & $13.3(9.8-16.9)$ & $13.3(9.4-17.1)$ & $36.5(32.6-40.3)$ & $33.2(29.3-37.1)$ \\
\hline \multicolumn{5}{|l|}{ Mother education } \\
\hline Elementary & $8.5(5.6-11.4)$ & $7.1(4.2-9.9)$ & $6.0(4.1-7.8)$ & $6.9(4.8-8.9)$ \\
\hline Lower secondary & $25.8(21.2-30.3)$ & $26.4(21.4-31.3)$ & $26.1(22.7-29.5)$ & $29.3(25.5-33.0)$ \\
\hline Upper secondary & $42.7(37.6-47.8)$ & $40.8(35.3-31.3)$ & $31.4(27.8-35.1)$ & $32.8(28.9-36.7)$ \\
\hline University degree & $23.0(18.7-27.4)$ & $25.7(20.8-30.6)$ & $36.5(32.7-40.2)$ & $31.0(27.2-34.9)$ \\
\hline \multicolumn{5}{|l|}{ Father education } \\
\hline Elementary & $8.3(5.5-11.2)$ & $7.0(4.1-9.8)$ & $6.1(4.2-8.0)$ & $5.2(3.4-7.1)$ \\
\hline Lower secondary & $28.3(23.7-33.0)$ & $23.5(18.7-28.3)$ & $29.7(26.1-33.4)$ & $32.0(28.1-35.8)$ \\
\hline Upper secondary & $44.4(39.3-49.6)$ & $48.7(43.0-54.4)$ & $25.8(22.3-29.3)$ & $30.2(26.3-34.0)$ \\
\hline University degree & $18.9(14.8-23.0)$ & $20.9(16.3-25.5)$ & $38.4(34.6-42.3)$ & $32.7(28.8-36.6)$ \\
\hline
\end{tabular}

BRACAH: Brazilian Cardiovascular Adolescent Health Study; HELENA: Healthy Lifestyle in Europe by Nutrition in Adolescence Study; n: Sample Size;

CI: Confidence Interval. 
Table 2. Clusters of energy balance related behaviors according to socioeconomic status and parental education in the Brazilian Cardiovascular Adolescent Health (BRACAH) and the Healthy Lifestyle in Europe by Nutrition in Adolescence (HELENA) studies, Brazil and Europe, 2007.

\begin{tabular}{|c|c|c|c|c|c|c|c|}
\hline \multirow{2}{*}{$\begin{array}{l}\text { Study } \\
\text { Clusters of EBRB }\end{array}$} & \multicolumn{4}{|c|}{ BRACAH GIRLS } & \multicolumn{3}{|c|}{ BRACAH BOYS } \\
\hline & $\begin{array}{l}\text { 1. Sedentary } \\
\%(95 \% \text { CI })\end{array}$ & $\begin{array}{l}\text { 2. Active } \\
\%(95 \% \mathrm{CI})\end{array}$ & $\begin{array}{c}\text { 3. Unhealthy Eating } \\
\%(95 \% \text { CI })\end{array}$ & $\begin{array}{l}\text { 4. Healthy Eating } \\
\%(95 \% \text { CI })\end{array}$ & $\begin{array}{l}\text { 1. Sedentary } \\
\%(95 \% \text { CI) }\end{array}$ & $\begin{array}{l}\text { 2. Active } \\
\%(95 \% \mathrm{CI})\end{array}$ & $\begin{array}{l}\text { 3. Healthy Eating } \\
\%(95 \% \text { CI) }\end{array}$ \\
\hline \multicolumn{8}{|l|}{ Socioeconomic status } \\
\hline Low & $7.5(1.1-13.8)$ & $11.3(2.7-20.0)$ & $8.3(3.6-13.0)$ & $11.1(5.4-16.9)$ & $3.6(0.5-6.7)$ & $2.9(0.11-7.1)$ & $6.7(01.9-11.5)$ \\
\hline Medium & $77.6(67.5-87.7)$ & $75.5(63.7-87.2)$ & $80.5(73.7-87.2)$ & $75.2(67.3-83.1)$ & $85.0(79.0-91.0)$ & $82.1(72.8-91.4)$ & $77.1(69.0-85.2)$ \\
\hline High & $14.9(6.3-23.6)$ & $13.2(4.0-22.4)$ & $11.3(5.9-16.7)$ & $13.7(7.4-19.9)$ & $11.4(06.1-16.7)$ & $14.9(06.3-23.6)$ & $16.2(09.1-23.3)$ \\
\hline \multicolumn{8}{|l|}{ Mother education } \\
\hline Elementary & $6.2(0.3-12.1)$ & $9.4(1.5-17.4)$ & $9.1(4.2-14.0)$ & $8.7(3.5-13.9)$ & $6.5(2.4-10.6)$ & $7.5(1.1-13.8)$ & $7.6(2.5-12.7)$ \\
\hline Lower secondary & $18.5(8.9-28.0)$ & $26.4(14.4-38.4)$ & $28.0(20.3-35.7)$ & $27.0(18.8-35.1)$ & $25.9(18.6-33.2)$ & $28.4(17.4-39.2)$ & $25.7(17.3-34.1)$ \\
\hline Upper secondary & $55.4(43.2-67.6)$ & $50.9(37.3-64.6)$ & $41.7(33.2-50.1)$ & $33.0(24.4-41.7)$ & $48.9(40.5-57.3)$ & $32.8(21.5-44.2)$ & $35.2(26.0-44.4)$ \\
\hline University degree & $20.0(10.2-29.8)$ & $13.2(34.0-22.4)$ & $21.2(14.2-28.2)$ & $31.3(22.8-39.8)$ & $18.7(12.2-25.2)$ & $31.3(20.1-42.6)$ & $31.4(22.5-40.4)$ \\
\hline \multicolumn{8}{|l|}{ Father education } \\
\hline Elementary & $3.0(-1.1-7.1)$ & $13.5(4.1-22.9)$ & $10.1(4.8-15.3)$ & $7.1(2.3-12.0)$ & $5.9(1.9-9.9)$ & $9.4(2.1-16.6)$ & $6.9(1.9-11.8)$ \\
\hline Lower secondary & $20.9(11.1-30.7)$ & $26.9(14.7-39.1)$ & $32.6(24.4-40.7)$ & $28.6(20.1-37.0)$ & $26.5(19.0-39.9)$ & $23.4(12.9-33.9)$ & $19.6(11.8-27.4)$ \\
\hline Upper secondary & $52.2(40.2-64.3)$ & $48.1(34.3-61.8)$ & $40.3(31.8-48.8)$ & $42.9(33.6-52.1)$ & $50.0(41.5-58.5)$ & $48.4(36.0-60.8)$ & $47.1(37.3-56.8)$ \\
\hline University degree & $23.9(13.6-34.2)$ & $11.5(2.7-20.3)$ & $17.1(10.5-23.6)$ & $21.4(13.8-29.1)$ & $17.7(11.2-24.1)$ & $18.8(9.1-28.4)$ & $26.5(17.8-35.1)$ \\
\hline Study & \multicolumn{4}{|c|}{ HELENA GIRLS } & \multicolumn{3}{|c|}{ HELENA BOYS } \\
\hline Clusters of EBRB & $\begin{array}{l}\text { 1. Sedentary } \\
\%(95 \% \text { CI })\end{array}$ & $\begin{array}{l}\text { 2. Active } \\
\%(95 \% \mathrm{CI})\end{array}$ & $\begin{array}{l}\text { 3. Unhealthy Eating } \\
\%(95 \% \text { CI })\end{array}$ & $\begin{array}{l}\text { 4. Healthy Eating } \\
\%(95 \% \text { CI) }\end{array}$ & $\begin{array}{l}\text { 1. Sedentary } \\
\%(95 \% \mathrm{CI})\end{array}$ & $\begin{array}{l}\text { 2. Healthy } \\
\% \text { (95\% CI) }\end{array}$ & $\begin{array}{c}\text { 3. Unhealthy Eating } \\
\%(95 \% \text { CI })\end{array}$ \\
\hline \multicolumn{8}{|l|}{ Socioeconomic status } \\
\hline Low & $14.5(8.6-20.4)$ & $15.0(8.2-21.8)$ & $11.5(6.9-16.2)$ & $6.9(3.6-10.1)$ & $15.6(9.6-21.6)$ & $11.6(7.6-15.5)$ & $7.0(3.5-10.6)$ \\
\hline Medium & $58.7(50.4-67.0)$ & $56.1(46.6-65.5)$ & $51.7(44.4-58.9)$ & $50.2(43.8-56.7)$ & $56.7(48.5-65.0)$ & $55.4(49.2-61.6)$ & $58.5(51.6-65.4)$ \\
\hline High & $26.8(19.4-34.2)$ & $29.0(20.3-37.6)$ & $36.8(29.8-43.9)$ & $42.9(36.5-49.3)^{*}$ & $27.7(20.2-35.1)$ & $33.1(27.2-38.9)$ & $34.5(27.9-41.1)^{*}$ \\
\hline \multicolumn{8}{|l|}{ Mother education } \\
\hline Elementary & $9.9(4.7-15.0)$ & $5.9(1.3-10.6)$ & $6.8(3.1-10.6)$ & $3.1(0.8-5.3)$ & $11.0(5.7-16.3)$ & $5.8(2.8-8.7)$ & $5.3(2.1-8.4)$ \\
\hline Lower secondary & $33.3(25.3-41.4)$ & $24.8(16.3-33.2)$ & $27.8(21.2-34.5)$ & $21.2(15.8-26.5)$ & $41.9(33.6-50.3)$ & $19.4(14.4-24.4)$ & $32.8(26.1-39.5)$ \\
\hline Upper secondary & $34.9(26.7-43.0)$ & $37.6(28.1-47.1)$ & $30.1(23.3-36.9)$ & $27.8(21.9-33.6)$ & $25.0(17.7-32.3)$ & $38.0(31.9-44.2)^{*}$ & $31.7(25.1-38.4)$ \\
\hline University degree & $22.0(14.9-29.1)$ & $31.7(22.6-40.8)$ & $35.2(28.1-42.3)$ & $48.0(41.5-54.5)^{*}$ & $22.1(15.0-29.0)$ & $36.8(30.7-42.9)^{*}$ & $30.2(23.6-36.7)$ \\
\hline \multicolumn{8}{|l|}{ Father education } \\
\hline Elementary & $9.4(4.3-14.6)$ & $4.2(0.1-8.3)$ & $8.9(4.6-13.2)$ & $2.8(0.6-4.9)$ & $6.25(2.0-10.5)$ & $5.0(2.2-7.8)$ & $4.8(1.7-78.1)$ \\
\hline Lower secondary & $33.9(25.6-42.1)$ & $29.5(20.2-38.7)$ & $34.9(27.7-42.2)$ & $23.4(17.8-29.0)$ & $45.3(36.6-54.0)$ & $21.7(16.4-26.9)$ & $36.0(29.1-42.9)$ \\
\hline Upper secondary & $30.7(22.6-38.8)$ & $25.3(16.5-34.1)$ & $23.7(17.3-30.1)$ & $24.8(19.0-30.5)$ & $25.0(17.5-32.5)$ & $33.3(27.3-39.3)$ & $29.6(23.1-36.2)$ \\
\hline University degree & $26.0(18.3-33.7)$ & $41.1(31.1-51.0)$ & $32.5(25.4-39.6)$ & $49.1(42.4-55.7)^{*}$ & $23.4(16.1-30.8)$ & $40.0(33.8-46.2)$ & $29.6(23.1-36.2)$ \\
\hline
\end{tabular}

BRACAH: Brazilian Cardiovascular Adolescent Health Study; HELENA: Healthy Lifestyle in Europe by Nutrition in Adolescence Study; EBRB: Energy Balance Related Behaviors; \%: Prevalence.CI: Confidence Interval.
Con formato: Fuente: Times New Roman, 10 pto Con formato: Fuente: Times New Roman 
International Journal of Public Health

How do energy balance-related behaviors cluster in adolescents?

Table 3. Nominal $p$-values of clusters of energy balance related behaviors according to socioeconomic status and parental education in the Brazilian Cardiovascular Adolescent Health (BRACAH) and the Healthy Lifestyle in Europe by Nutrition in Adolescence (HELENA) studies ${ }_{-2}$. Brazil and Europe, 2007.

\begin{tabular}{|c|c|c|c|c|c|c|c|}
\hline \multirow{2}{*}{$\begin{array}{l}\text { Study } \\
\text { Clusters of EBRB }\end{array}$} & \multicolumn{4}{|c|}{ BRACAH GIRLS } & \multicolumn{3}{|c|}{ BRACAH BOYS } \\
\hline & 1. Sedentary & $\begin{array}{l}\text { 2. Active } \\
p \text {-value }\end{array}$ & $\begin{array}{c}\text { 3. Unhealthy Eating } \\
p \text {-value }\end{array}$ & $\begin{array}{l}\text { 4. Healthy Eating } \\
p \text {-value }\end{array}$ & 1. Sedentary & $\begin{array}{l}\text { 2. Active } \\
p \text {-value }\end{array}$ & $\begin{array}{c}\text { 3. Healthy Eating } \\
p \text {-value }\end{array}$ \\
\hline \multicolumn{8}{|c|}{ Socioeconomic status } \\
\hline Low & Ref. & Ref. & Ref. & Ref. & Ref. & Ref. & Ref. \\
\hline Medium & Ref. & 0.488 & 0.906 & 0.439 & Ref. & 0.865 & 0.232 \\
\hline High & Ref. & 0.490 & 0.571 & 0.464 & Ref. & 0.631 & 0.686 \\
\hline Mother education & & & & & Ref. & & \\
\hline Elementary & Ref. & Ref. & Ref. & Ref. & Ref. & Ref. & Ref. \\
\hline Lower secondary & Ref. & 0.929 & 0.967 & 0.962 & Ref. & 0.935 & 0.757 \\
\hline Upper secondary & Ref. & 0.476 & 0.273 & 0.175 & Ref. & 0.375 & 0.352 \\
\hline University degree & Ref. & 0.303 & 0.620 & 0.879 & Ref. & 0.553 & 0.519 \\
\hline Father education & & & & & Ref. & & \\
\hline Elementary & Ref. & Ref. & Ref. & Ref. & Ref. & Ref. & Ref. \\
\hline Lower secondary & Ref. & 0.158 & 0.346 & 0.512 & Ref. & 0.344 & 0.440 \\
\hline Upper secondary & Ref. & 0.060 & 0.062 & 0.192 & Ref. & 0.392 & 0.697 \\
\hline University degree & Ref. & 0.017 & 0.061 & 0.251 & Ref. & 0.530 & 0.669 \\
\hline Study & \multicolumn{4}{|c|}{ HELENA GIRLS } & \multicolumn{3}{|c|}{ HELENA BOYS } \\
\hline Clusters of EBRB & 1. Sedentary & $\begin{array}{l}\text { 2. Active } \\
P \text {-value }\end{array}$ & $\begin{array}{c}\text { 3. Unhealthy Eating } \\
P \text {-value }\end{array}$ & $\begin{array}{l}\text { 4. Healthy Eating } \\
P \text {-value }\end{array}$ & $\begin{array}{l}\text { 1. Sedentary } \\
P \text {-value }\end{array}$ & $\begin{array}{l}\text { 2. Healthy } \\
P \text {-value }\end{array}$ & $\begin{array}{c}\text { 3. Unhealthy Eating } \\
P \text {-value }\end{array}$ \\
\hline \multicolumn{8}{|c|}{ Socioeconomic status } \\
\hline Low & Ref. & Ref. & Ref. & Ref. & Ref. & Ref. & Ref. \\
\hline Medium & Ref. & 0.773 & 0.838 & 0.106 & Ref. & 0.382 & 0.025 \\
\hline High & Ref. & 0.145 & 0.911 & $0.002 *$ & Ref. & 0.163 & $0.010^{*}$ \\
\hline \multicolumn{8}{|l|}{ Mother education } \\
\hline Elementary & Ref. & Ref. & Ref. & Ref. & Ref. & Ref. & Ref. \\
\hline Lower secondary & Ref. & 0.677 & 0.707 & 0.169 & Ref. & 0.768 & 0.274 \\
\hline Upper secondary & Ref. & 0.621 & 0.281 & 0.066 & Ref. & $0.012^{*}$ & 0.035 \\
\hline University degree & Ref. & 0.067 & 0.117 & $<0.001$ & Ref. & $0.007^{*}$ & 0.025 \\
\hline \multicolumn{8}{|l|}{ Father education } \\
\hline Elementary & Ref. & Ref. & Ref. & Ref. & Ref. & Ref. & Ref. \\
\hline Lower secondary & Ref. & 0.831 & 0.285 & 0.110 & Ref. & 0.298 & 0.936 \\
\hline Upper secondary & Ref. & 0.659 & 0.333 & 0.060 & Ref. & 0.309 & 0.408 \\
\hline University degree & Ref. & 0.518 & 0.043 & $0.001 *$ & Ref. & 0.131 & 0.345 \\
\hline
\end{tabular}

Significant associations based on multilevel regression test and Bonferroni Correction ( $P<0.017$ for Socioeconomic Status; $P<0.013$ for Parental Education).

BRACAH: Brazilian Cardiovascular Adolescent Health Study; HELENA: Healthy Lifestyle in Europe by Nutrition in Adolescence Study; EBRB: Energy Balance Related Behaviors; Ref: Reference. 